\title{
Image Retrieval by Using Non-Subsampled Shearlet Transform and Krawtchouk Moment Invariants
}

\author{
Cheng Wan, Yiquan Wu \\ 29 Yudao St., Nanjing 210016, China
}

\begin{abstract}
In this paper, we use non-subsampled shearlet transform (NSST) and Krawtchouk Moment Invariants (KMI) to realize image retrieval based on texture and shape features. Shearlet is a new sparse representation tool of multidimensional function, which provides a simple and efficient mathematical framework. We decompose the images by NSST. The directional subband coefficients are modeled by Generalized Gaussian Distribution (GGD). The distribution parameters are used to build texture feature vectorswhich are measured by Kullback-Leibler distance (KLD). Meanwhile, low-order KMI are employed to extract shape features which are measured by Euclidean distance (ED). Finally, the image retrieval is achieved based on weighted distance measurement. Experimental results show the proposed retrieval system can obtain the highest retrieval rate comparing with the methods based on DWT, Contourlet, NSCT and DT-CWT.
\end{abstract}

\section{Introduction}

Retrieving similar image from an image database is a challenging task. Text based image retrieval systems (TBIR) were used from the 1970's. Searching images based on the content of the image is called Content Based Image Retrieval (CBIR) which uses different features of the image to search similar images from an image database. The CBIR appeared in the 1990's and it is suitable for both large and small size databases [1-3]. CBIR adopts feature extraction and similarity measurement for image retrieval process. Image content features such as colour, texture and shape, which are analyzed and extracted automatically by computer achieves the effective retrieval.

Texture can efficiently reflect the structural, directional, granularity, or regularity differences of diverse regions in a visual image. Therefore, texture features become one of the most efficient and effective models used in CBIR systems. Discrete wavelet transform can represent the texture features into some subband properties such as the subband energy, which was studied sufficiently at the early stage [4-6]. Later, Gabor wavelets and complex wavelets were developed to remove the limitation of the wavelet transform to capture more directional information in subbands. Contourlet transform $[7,8]$ is a new image analysis tool, which is anisotropic and has good directional selectivity. The sub-sampling operation is employed in the implementation of Contourlet transform. So it is not 
shift-invariant and sub-band spectrum aliasing phenomenon takes place, which weakens the directional selectivity of Contourlet transform. To overcome this disadvantage, Cunha et al. [9] proposed non-subsampled Contourlet transform (NSCT) which is an improved version of Contourlet transform]. But the computational efficiency of NSCT is low. In 2005, Labate et al. [10] proposed a new multi-scale geometric analysis tools: shearlet which is optimally sparse in representing images. The decomposition of shearlet is similar to Contourlet transform, but an important advantage of shearlet over Contourlet transform is that there are no restrictions on the number of directions for the shearing. In addition, the inverse shearlet transform only requires a summation of the shearing filters rather than inverting a directional filter banks, thus the implementation of shearlet is more efficient computationally. Due to these advantages with the shearlet, it has been applied in many image processing fields, such as image denoising, edge detection, and image fusion. The shearlet transform also uses the subsampling operation, so it is not shift-invariant and it will produce pseudo-Gibbs phenomenon around the singular point. The non-subsampled shearlet transfor$\mathrm{m}$ [11] by cascading of non-subsampled pyramid filter banks and shearing filter banks has all the advantages of the shearlet transform and does not need the implementation of the sub-sampling operation. In wavelet domain, the detail subband coefficients of a texture image usually exhibit a striking non-Gaussian behavior, and their marginal distribution can be well characterized by the generalized Gaussian distribution (GGD) [12]. We use GGD to model the subbands of NSST and use maximum likelihood (ML) estimation to estimate the parameters of GGD.

On the other hand, image retrieval based on shape content remains a more difficult task than that based on other visual features [13-15]. Shape is one of the most basic and meaningful characteristics, shape descriptors should be invariant to translation, rotation and scaling changes of the object on the basis of distinguishing different objects. Moment has been widely used in pattern recognition applications to describe the geometrical characteristics of different objects, its calculation is related to all of the relevant pixels of the image or region, it can describe the global information, and Krawtchouk moment [16] proposed by Yap is a set of discrete orthogonal moments, there is no need for spatial normalization, hence the error in the computed Krawtchouk moments due to discretization is nonexistent, and it has smaller redundancy than other moments, at the same time Krawtchouk moment invariants is invariant to rotation, scaling, and translation of the image. Krawtchouk moment invariants as global shape features achieve image retrieval. We extract Krawtchouk moment invariants as global feature of the image, and only use low-order moments that can describe the shape information. High-order moments need great amount of calculation and only describe the details of an image.

In this paper, we decompose the image with NSST and compute the subband GGD parameters to represent the texture features. Kullback-Leibler distance (KLD) is used to measure the distance between texture features. Meanwhile, low-order Krawtchouk moment invariants are calculated to be the shape features 


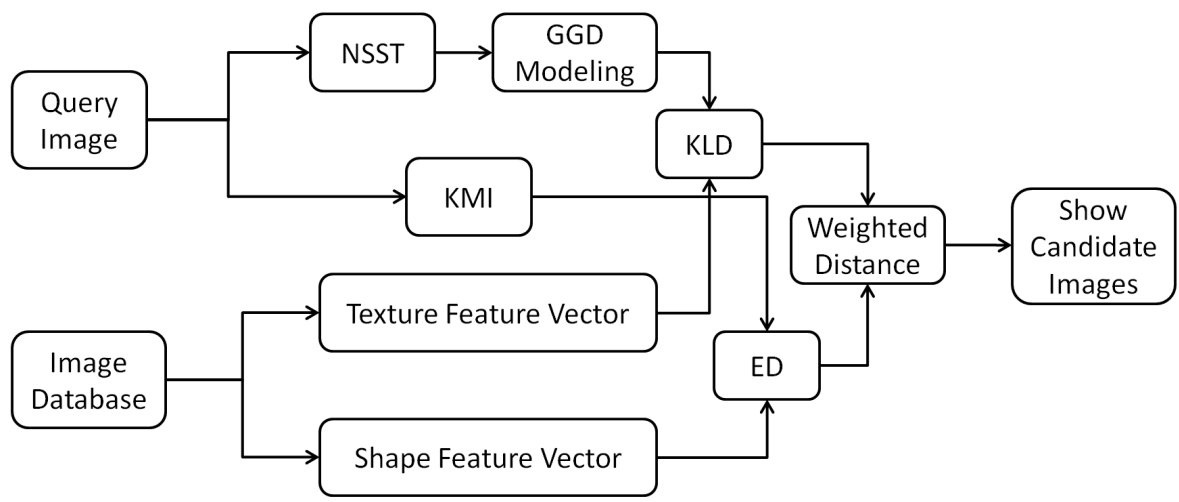

Fig. 1. Block diagram of the proposed image retrieval system

which are measured by Euclidean distance (ED). Finally, the weighted distance of KLD and ED is derived to measure the similarity between the query image and the database images. The effectiveness of the proposed retrieval scheme has been demonstrated by the experiments.

\section{Proposed image retrieval method}

The proposed image retrieval system works in two stages to collect the image information. The first stage measures the distribution of the textures in the image. The second stage measures the shape information from the image. Thus the collected information helps in enhanced retrieval. For each image in the database, its texture and shape feature vectors have been extracted and stored. The texture and shape features of the query image are calculated. To obtain the texture feature, the image is decomposed by NSST and the subband coefficients of NSST is modeled by Generalized Gaussian Density (GGD). The parameters of GGD are the texture feature of image. Meanwhile, we figure out the Krawtchouk moment invariants (KMI) of the image as the shape feature. Feature dissimilarity between the query image and the database images is measured by weighted distance of Kullback-Leibler distance (KLD) and Euclidean distance (ED). KLD is used to compute the distance between texture features and ED is for measuring the similarity of shape features. The system then outputs the most relevant images to the user in the ascending order of the weighted distance. The block diagram is given in Fig. 1 and the detailed explanation of every block is given in the following sections. 


\section{Texture feature extraction}

\subsection{Non-subsampled shearlet transform (NSST)}

In dimension $\mathrm{n}=2$, the affine systems of $\mathrm{ST}$ is defined as follows:

$$
\left\{\psi_{j, l, k}(x)=|\operatorname{det} \mathbf{A}|^{j / 2} \psi\left(\mathbf{B}^{l} \mathbf{A}^{j} x-k\right), j, l \in \mathbb{Z}, k \in \mathbb{Z}^{2}\right\}
$$

where $\psi$ is a collection of basis function and satisfies $\psi \in L^{2}\left(\mathbb{R}^{2}\right)$, A denotes the anisotropy matrix for multi-scale partitions, $\mathbf{B}$ is a shear matrix for directional analysis. $j, l, k$ are scale, direction and shift parameter respectively. $\mathbf{A}, \mathbf{B}$ are both $2 \times 2$ invertible matrices and $\operatorname{det}|\mathbf{B}|=1$. For each $a>0$ and $b \in \mathbf{R}$, the matrices of $\mathbf{A}$ and $\mathbf{B}$ are represented as:

$$
\mathbf{A}=\left(\begin{array}{cc}
a & 0 \\
0 & \sqrt{a}
\end{array}\right), \mathbf{B}=\left(\begin{array}{ll}
1 & b \\
0 & 1
\end{array}\right)
$$

These two matrices are important roles in the process of shearlet transform. The former dominates the scaling of shearlet, and the latter controls the orientation of shearlet. When $\mathrm{a}=4, \mathrm{~s}=1,(2)$ is written as follows:

$$
\mathbf{A}=\left(\begin{array}{ll}
4 & 0 \\
0 & 2
\end{array}\right), \mathbf{B}=\left(\begin{array}{ll}
1 & 1 \\
0 & 1
\end{array}\right)
$$

For any $\forall \xi=\left(\xi_{1}, \xi_{2}\right) \in \widehat{\mathbb{R}}^{2}, \xi_{1} \neq 0, \psi^{(0)}$ for ST can be described as:

$$
\widehat{\psi}^{(0)}(\xi)=\widehat{\psi}^{(0)}\left(\xi_{1}, \xi_{2}\right)=\widehat{\psi}_{1}\left(\xi_{1}\right) \widehat{\psi}_{2}\left(\frac{\xi_{2}}{\xi_{1}}\right)
$$

Here, $\widehat{\psi}$ is the Fourier transform of $\psi, \operatorname{supp} \widehat{\psi}_{1} \subset\left[-\frac{1}{2},-\frac{1}{16}\right] \cup\left[\frac{1}{16}, \frac{1}{2}\right] \operatorname{supp} \widehat{\psi}_{2} \subset$ $[-1,1]$.

$$
\begin{aligned}
& \text { Assume that } \\
& \sum_{j \geq 0}\left|\widehat{\psi}_{1}\left(2^{-2 j} \omega\right)\right|^{2}=1, \quad|\omega| \geq \frac{1}{8} \\
& \sum_{l=-2^{j}}^{2^{j}-1}\left|\widehat{\psi}_{2}\left(2^{-2 j} \omega-l\right)\right|=1, \quad|\omega| \leq 1, j \geq 0
\end{aligned}
$$

we can see $\psi_{j, l, k}(x)$ has the frequency support as follows.

$$
\operatorname{supp} \widehat{\psi}_{j, l, k}^{(0)} \subset\left\{\left(\xi_{1}, \xi_{2}\right): \xi_{1} \in\left[-2^{2 j-1},-2^{2 j-4}\right] \cup\left[2^{2 j-4}, 2^{2 j-1}\right],\left|\frac{\xi_{2}}{\xi_{1}}+l 2^{-j}\right| \leq 2^{-j}\right\}
$$

Hence, $\widehat{\psi}_{j, l, k}$ is supported on a pair of trapeziform zones, whose sizes all approximate to $2^{2 j} \times 2^{j}$. 
The shearlet transform has the following main properties: well localizing, parabolic scaling, highly directional sensitivity, spatially localizing and optimally sparse. The non-subsampled shearlet transform (NSST), which combined the non-subsampled Laplacian pyramid transform with several different combinations of the shearing filters, is the shift-invariant version of the shearlet transfor$m$. The NSST differs from the shearlet transform in that the NSST eliminates the down-samplers and up-samplers. The NSST is a fully shift-invariant, multiscale, and multi-direction expansion. Consequently, introduction of NSST into image retrieval could make use of the good characters of NSST in effectively extracting features from original images.

\subsection{GGD probability density function (PDF)}

The PDF of a zero-mean generalized Gaussian distribution (GGD) is defined as:

$$
p\left(x ; \sigma^{2}, \beta\right)=\frac{\beta \eta(\sigma, \beta)}{2 \Gamma(1 / \beta)} \exp \left\{-[\eta(\sigma, \beta)|x|]^{\beta}\right\}
$$

where $\sigma^{2}$ and $\beta$ denote the variance and the shape parameter of the distribution respectively. $\Gamma$ is the gamma function given by $\Gamma(x)=\int_{0}^{\infty} t^{x-1} e^{-t} d t, x>0$, and

$$
\log \Gamma(x)=-\gamma x-\log (x)+\sum_{k=1}^{\infty}\left[\frac{x}{k}-\log \left(1+\frac{x}{k}\right)\right]
$$

where $\gamma=0.577$ denotes the Euler constant. In addition, $\eta(\sigma, \beta)$ is given by

$$
\eta(\sigma, \beta)=\frac{1}{\sigma}\left[\frac{\Gamma(3 / \beta)}{\Gamma(1 / \beta)}\right]^{1 / 2}
$$

Here, the smaller $\beta$ corresponds to the sharper distribution, while the bigger $\beta$ represents the flatter distribution.

\subsection{GGD modeling of NSST direction subband coefficients}

Given $N$ samples $\mathbf{x}=\left(x_{1}, x_{2}, \cdots, x_{N}\right)$ with zero mean PDF of GGD, The ML method gives the estimates of parameters $\sigma$ and $\beta$ as follows.

$$
\begin{aligned}
& \hat{\sigma}=\left[\frac{\Gamma(3 / \beta)}{\Gamma(1 / \beta)}\right]^{1 / 2}\left[\frac{\hat{\beta}}{N} \sum_{i=1}^{N}\left|x_{i}\right|^{\hat{\beta}}\right]^{1 / \hat{\beta}} \\
& 1+\frac{\Psi(1 / \hat{\beta})}{\hat{\beta}}-\frac{\sum_{i=1}^{N}\left|x_{i}\right|^{\hat{\beta}} \log \left|x_{i}\right|}{\sum_{i=1}^{N}\left|x_{i}\right|^{\hat{\beta}}}+\frac{\log \left(\frac{\hat{\beta}}{N} \sum_{i=1}^{N}\left|x_{i}\right|^{\hat{\beta}}\right)}{\hat{\beta}}=0
\end{aligned}
$$


where the digamma function is given by $\Psi(x)=\frac{d \log (\Gamma(x))}{d x}$. For the transcendental equation (12), $\hat{\beta}$ can be solved numerically, and then $\hat{\sigma}$ is obtained from (11). Therefore, it is sufficient to obtain the estimation of $\hat{\beta}$. [6] used the NewtonRaphson method with an initial value $E[|x|] / \sigma$ from the moment estimation to estimate $\hat{\beta}$.

In this paper, we use a zero mean GGD model to characterize the NSST subband coefficients. The GGD parameters, $\sigma^{2}$ and $\beta$, are extracted as image texture features using ML method. Each subband corresponds one pair of $\left\{\sigma^{2}, \beta\right\}$. Assume NSST gives $n$ subbands, we can derive $2 n$ parameters of texture feature. The texture feature vector is described as: $\mathbf{V}_{t}=\left[\sigma_{1}^{2}, \beta_{1}, \sigma_{2}^{2}, \beta_{2}, \cdots, \sigma_{n}^{2}, \beta_{n}\right]$.

\section{Shape feature extraction}

\subsection{Krawtchouk moment invariants (KMI)}

The Krawtchouk moments are a set of orthogonal moments formed by using the discrete classical Krawtchouk polynomials as the basis function set. Because the basis polynomial is discrete, there is no need for spatial normalization, hence, the error in the computed Krawtchouk moments due to discretization is nonexistent. The $n$-th order Krawtchouk polynomial [16] is defined as follows.

$$
K_{n}(x ; p, N)=\sum_{k=0}^{n} a_{k, n, p} x^{k}={ }_{2} F_{1}\left(-n,-x ;-N ; \frac{1}{p}\right)
$$

where $x, n=0,1,2, \cdots, N, N>0, p \in(0,1)$. The hypergeometric function ${ }_{2} F_{1}(a, b ; c ; z)=\sum_{k=0}^{n} \frac{(a)_{k}(b)_{k}}{(c)_{k}} \frac{z^{k}}{k !} \cdot(a)_{k}$ is the Pochhammer symbol given by

$$
(a)_{k}=a(a+1) \cdots(a+k-1) .
$$

In order to achieve numerical stability, a set of weighted Krawtchouk polynomials $\bar{K}_{n}(x ; p, N)$ is defined by

$$
\bar{K}_{n}(x ; p, N)=K_{n}(x ; p, N) \sqrt{\frac{w(x ; p, N)}{\rho(n ; p, N)}}
$$

where $w(x ; p, N)=\left(\begin{array}{c}N \\ x\end{array}\right) p^{x}(1-p)^{N-x}, \rho(n ; p, N)=(-1)^{n}\left(\frac{1-p}{p}\right)^{n} \frac{n !}{(-N)_{n}}$.

The Krawtchouk moments of order $(n+m)$ for an $M_{1} \times M_{2}$ image with intensity function $f(x)$ is defined as

$$
Q_{n m}=\sum_{x=0}^{M_{1}-1} \sum_{y=0}^{M_{2}-1} \bar{K}_{n}(x) \bar{K}_{m}(y) \tilde{f}(x, y)
$$

where $\tilde{f}(x, y)=\left[w\left(x ; p, M_{1}\right) w\left(y ; q, M_{2}\right)\right]^{-(1 / 2)} f(x, y), \bar{K}_{n}(x)=\bar{K}_{n}\left(x ; p, M_{1}-1\right)$, $\bar{K}_{m}(y)=\bar{K}_{m}\left(y ; q, M_{2}-1\right)$. 
The set of Krawtchouk moment invariants (KMI) is defined as follows.

$$
\tilde{Q}_{n m}=[\rho(n) \rho(m)]^{-\frac{1}{2}} \sum_{i=0}^{n} \sum_{j=0}^{m} a_{i, n, p} a_{j, m, q} \tilde{v}_{i j}
$$

where $\rho(n)=\rho\left(n ; p, M_{1}\right), \rho(m)=\rho\left(m ; q, M_{2}\right)$,

$$
\tilde{v}_{i j}=\sum_{i=0}^{n} \sum_{j=0}^{m}\left(\begin{array}{c}
n \\
i
\end{array}\right)\left(\begin{array}{c}
m \\
q
\end{array}\right)\left(\frac{N^{2}}{2}\right)^{(i+j) / 2+1}\left(\frac{N}{2}\right)^{(n+m-i-j)} v_{i j} .
$$

$v_{i j}$ is the standard set of geometric moment invariants.

\subsection{Shape feature extraction by KMI}

The Krawtchouk moment invariants are rotation, scale and translation invariant, hence in this paper shape features of images are extracted with KMI. The shape feature vector is represented as: $\mathbf{f}=\left[\tilde{Q}_{00}, \tilde{Q}_{01}, \tilde{Q}_{10}, \cdots, \tilde{Q}_{n m}\right]$. Normalize the Krawtchouk moment invariants:

$$
\bar{Q}_{i j}=\frac{\tilde{Q}_{i j}-\mu_{\mathbf{f}}}{\sigma_{\mathbf{f}}}
$$

where $\mu_{\mathbf{f}}$ and $\sigma_{\mathbf{f}}$ are mean and standard deviation of $\mathbf{f}$ respectively. Then the normalized shape feature vector is written as:

$$
\mathbf{V}_{s}=\left[\bar{Q}_{00}, \bar{Q}_{01}, \bar{Q}_{10}, \cdots, \bar{Q}_{n m}\right]
$$

\section{Similarity measurement}

\subsection{Distance between texture vectors}

The PDF of each NSST subband coefficients can be described by parameter $\sigma^{2}$ and $\beta$ in the GGD model. Here, the distance between the GGD parameter pairs is calculated with the Kullback-Leibler distance (KLD):

$$
D\left(p\left(. ; \sigma^{2}, \beta\right) \| p\left(. ; \sigma^{\prime 2}, \beta^{\prime}\right)\right)=\log \left(\frac{\beta \sigma^{\prime 2} \Gamma\left(1 / \beta^{\prime}\right)}{\beta^{\prime} \sigma^{2} \Gamma(1 / \beta)}\right)+\left(\frac{\sigma^{2}}{\sigma^{\prime 2}}\right)^{\beta^{\prime}} \frac{\Gamma\left(\left(\beta^{\prime}+1\right) / \beta\right)}{\Gamma(1 / \beta)}-\frac{1}{\beta}
$$

The similarity measurement between two NSST subbands can be figured out effectively by the PDF parameters. Meanwhile, the NSST coefficients in different subbands are independent, therefore, the overall distance between two images is the sum of all the KLDs across the NSST subbands. The texture feature distance between the query image and the database image is represented as follows.

$$
D\left(\mathbf{V}_{t}, \mathbf{V}_{t}^{\prime}\right)=\sum_{i=1}^{S} D\left(p\left(. ; \sigma_{i}^{2}, \beta_{i}\right) \| p\left(. ; \sigma_{i}^{\prime 2}, \beta_{i}^{\prime}\right)\right)
$$

where $S$ is the number of the subbands. There is no need for normalization on texture feature vectors in this method of similarity measurement. 


\subsection{Distance between shape vectors}

The similarity measurement between the shape feature vectors is selected to be Euclidean distance, which is the most common distance measurement and is defined as follows.

$$
D\left(\mathbf{V}_{s}, \mathbf{V}_{s}^{\prime}\right)=\left(\sum_{i=1}^{K}\left(V_{s i}-V_{s i}^{\prime}\right)^{2}\right)^{\frac{1}{2}}
$$

where $\mathbf{V}_{s}$ is the shape feature vector of the query image, $\mathbf{V}_{s}^{\prime}$ is the shape feature vector of the image in the database, and $K$ is the number of vector elements.

\subsection{Weighted distance measurement}

The final distance between the query image $\mathbf{I}$ and the database image $\mathbf{I}^{\prime}$ is defined by the weighted distance formula as follows.

$$
D\left(\mathbf{I}, \mathbf{I}^{\prime}\right)=w D\left(\mathbf{V}_{t}, \mathbf{V}_{t}^{\prime}\right)+(1-w) D\left(\mathbf{V}_{s}, \mathbf{V}_{s}^{\prime}\right)
$$

where $w$ is the weighted coefficient and $0 \leq w \leq 1$. We use a minimum distance criterion and sort the database images for each query.

\section{Experimental studies}

By extracting the texture features and shape features, and measuring the weighted distance developed above, we proposed a NSST-KML-based retrieval system. The retrieval task is to search the top $N$ images that are similar to one query image within a large database of total $M$ unlabeled images. In the proposed system, each image is decomposed into multiple directional subbands. Then, we use the GGD to model the subband coefficients. Parameters $\left\{\sigma^{2}, \beta\right\}$ are derived with ML method, which are the extracted image texture features. The Kullback-Leibler distance (KLD) between the query image and each database image is measured. Meanwhile, the low-order Krawtchouk moment invariants are extracted to be the image shape features. Calculate the Euclidean distance (ED) between the query image and each database image. Finally, the weighted distance of KLD and ED is measured. The top $N$ database images that have the smallest weighted distance are retrieved.

To validate the performance of the proposed retrieval system, we use 40 classes obtained from VisTex database. A test database of 640 texture images is constructed by dividing each $512 \times 512$ image into 16 non-overlapping $128 \times 128$ subimages. In retrieval experiments, the query image is taken randomly from 640 subimages, and relevant candidate images are the other 15 subimages from the same class. We show 3 sets of experiments and analyze the results. First, we compare the mean-variance-based method, GGD-based method and GGDKMI-based method across 5 transform: DWT, Contourlet, NSCT, DT-CWT and NSST. Secondly, we change the decomposition levels of NSST to evaluate the retrieval rate with the method mentioned above. Thirdly, we show the image retrieval result interfaces derived from the methods combining 5 different transforms with KMI respectively. 


\subsection{Comparison results from different transforms}

In this experiment, the images are all decomposed into 3 levels by DWT, Contourlet, NSCT, DT-CWT and NSST. For the proposed method, 6, 6, 10 directions are decomposed in the scales from coarser to finer for NSST, and the pyramid filter of NSST is set as maxflat. The shape feature vector built based on Krawtchouk moment invariants is constructed as: $\mathbf{V}_{s}=\left[\bar{Q}_{02}, \bar{Q}_{11}, \bar{Q}_{03}, \bar{Q}_{12}\right]$. The weighted coefficient $w$ is set to be 0.7 . The selection of parameters is according to the experimental results.

As shown in Table 1, we computed the overall recognition rate with 7 methods on the all 5 transforms. Overall recognition rate denotes the average recall for all the images in the database. $\{\mu\} \& \mathrm{ED}$ denotes only the mean of each subband is extracted to measure the similarity by Euclidean distance. $\sigma$ is the standard deviation of the subband. $\{\mu, \sigma\} \& \mathrm{ED}+\mathrm{KMI}$ is the method combining $\{\mu, \sigma\} \&$ ED with KMI. GGD\&KLD represents the retrieval system just extracts the texture features, which are modeled by GGD and measure the distance between the feature vectors with KL distance. GGD\&ED describes the similarity measurement of GGD parameters is obtained by ED. GGD\&KLD+KMI is the method combining GGD\&KLD with KMI.

From Table 1, we can see that:

(1) Method $\{\mu, \sigma\} \& \mathrm{ED}+\mathrm{K}$ is better than $\{\mu\} \& \mathrm{ED},\{\sigma\} \& \mathrm{ED}$ and $\{\mu, \sigma\} \& \mathrm{ED}$. The retrieval rate of $\{\mu\} \& \mathrm{ED}$ or $\{\sigma\} \& \mathrm{ED}$ is lower than $\{\mu, \sigma\} \& \mathrm{ED}$, since more features bring more information of images, which improves the efficiency of retrieval system. One the other hand, KMI provide information of image shape and global features, hence the hybrid method is better than the single feature method.

(2) Method GGD\&KLD is better than GGD\&ED. To GGD parameters $\left\{\sigma^{2}, \beta\right\}$, KLD is the more appropriate similarity measurement than ED.

(3) GGD\&KLD+KMI method is better than the second best method GGD\&KLD based on any transform. The reason is as same as the first summary. Texture features combining with shape features can describe an image more accurately. In additon, in order to extract shape features, we just use four low-order KMI, and the measurement of feature vectors is ED, hence the time cost on shape feature extraction and similarity measurement is quite low. However that brings better results.

(4) Methods based on NSST show the best results comparing with the methods on the other transforms. That demonstrates the good performance of NSST in comparison with other transforms.

\subsection{Results from different decomposition levels of NSST}

We also evaluate the performance of proposed method based on different decomposition levels of NSST. Table 2 shows the overall recognition rate with 7 methods on NSST of one scale with 6 directions, 2 scales with 6 directions respectively and three scales with $6,6,10$ directions. NSST of three decomposition levels gives the best results than that of one and two decomposition levels. The proposed method still derives best results comparing with the other methods. 
Table 1. Overall recognition rate(\%) with 7 methods across 5 different transforms

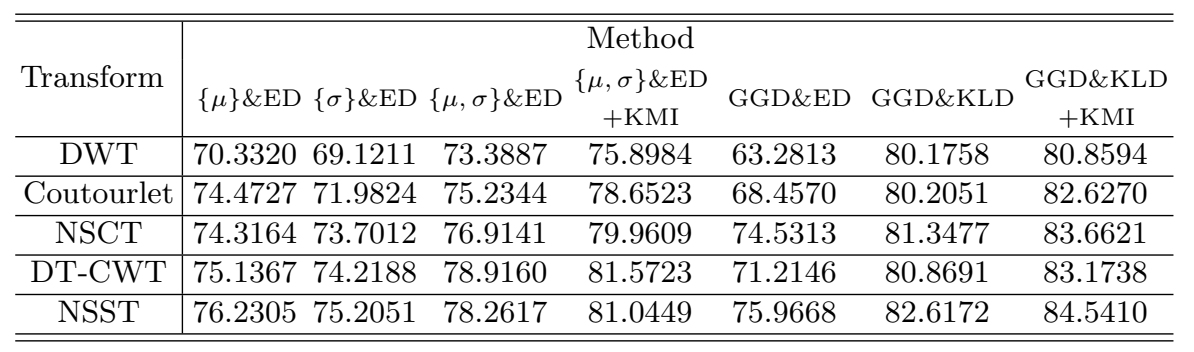

Table 2. Overall recognition rate(\%) with 7 methods based on different decomposition levels of NSST

\begin{tabular}{|c|c|c|c|c|c|c|c|}
\hline Scale & & & & Method & & & \\
\hline Direction & $\{\mu\} \& \mathrm{ED}$ & $\{\sigma\} \& \mathrm{ED}$ & $\{\mu, \sigma\} \& \mathrm{ED}$ & $\begin{array}{c}\{\mu, \sigma\} \& \mathrm{ED} \\
+\mathrm{KMI}\end{array}$ & GGD\&ED & GGD\&KLD & $\begin{array}{c}\text { GGD\&KLD } \\
+ \text { KMI }\end{array}$ \\
\hline $1[6]$ & 59.1602 & 58.8867 & 66.0742 & 71.2500 & 65.2930 & 71.2207 & 74.7949 \\
\hline $\left.2 \begin{array}{ll}6 & 6\end{array}\right]$ & 70.8008 & 69.7754 & 74.1992 & 77.7637 & 73.2617 & 79.5215 & 82.2754 \\
\hline $3\left[\begin{array}{llll}6 & 6 & 10\end{array}\right]$ & 76.2305 & 75.2051 & 78.2617 & 81.0449 & 75.9668 & 82.6172 & 84.5410 \\
\hline
\end{tabular}

\subsection{Image retrieval examples}

Four sets of comparison examples of GGD\&KLD+KMI method based on DWT, Coutourlet, NSCT, DT-CWT and NSST are shown in Fig. 2, Fig. 3, Fig. 4 and Fig. 5 respectively. Examples of retrieval results are computed from 640 texture images based on the VisTex database. In each example, the query image is on the top left corner and all other images are ranked in the order of similarity with the query image from left to right, top to bottom. The query images are Fabric, Food, Leaves and Tile respectively. The proposed method of GGD\&KLD+KMI based on NSST shows better retrieval performance than other methods.

\section{Conclusion}

In this paper, we proposed a new image retrieval system which is a hybrid method of NSST and KMI. The image was decomposed by NSST and GGD was used to fit the subband coefficients of NSST. The parameters of GGD were regarded as texture features which were measured by Kullback-Leibler distance. On the other hand, low-order Krawtchouk moment invariants were calculated to be the shape features which were measured by Euclidean distance. At last, the weighted distance of two kinds of distance was computed to measure the similarity between the query image and the database images. Three sets of experiments based on 7 methods and 5 different transforms showed the proposed method can obtain higher retrieval rate than other methods. 


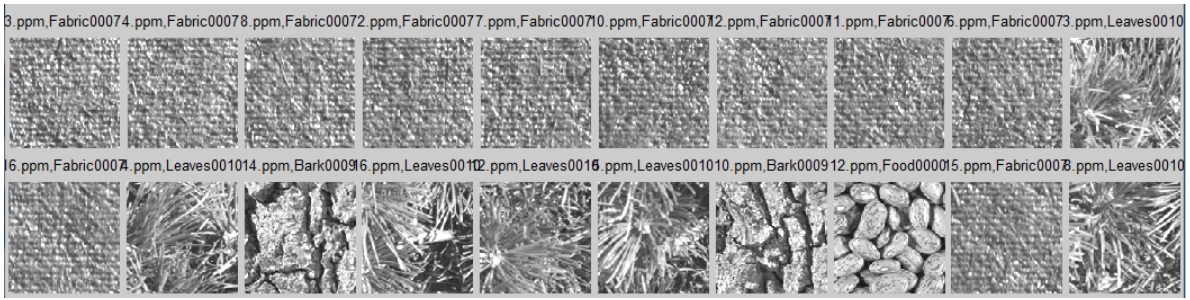

(a) GGD\&KLD+KMI method based on DWT

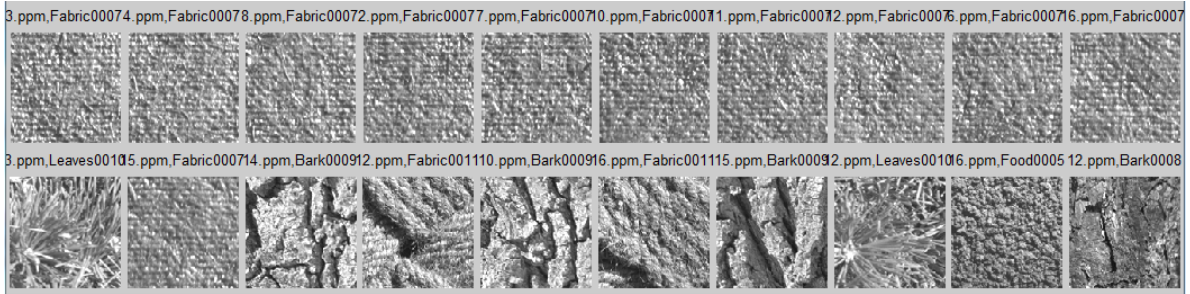

(b) GGD\&KLD+KMI method based on Coutourlet

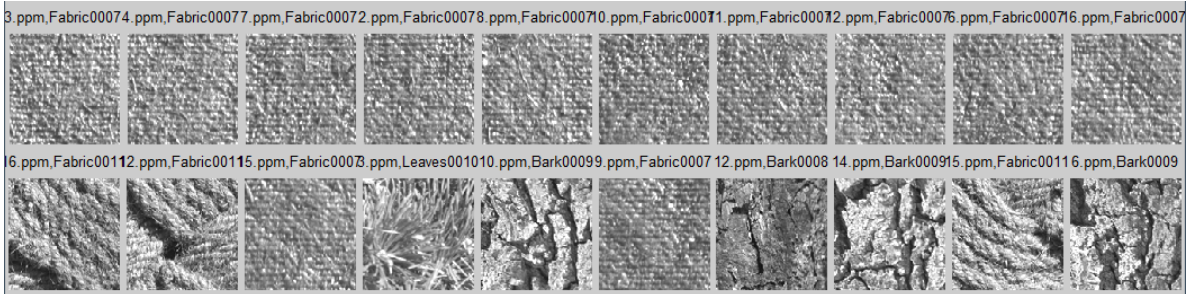

(c) GGD\&KLD+KMI method based on NSCT

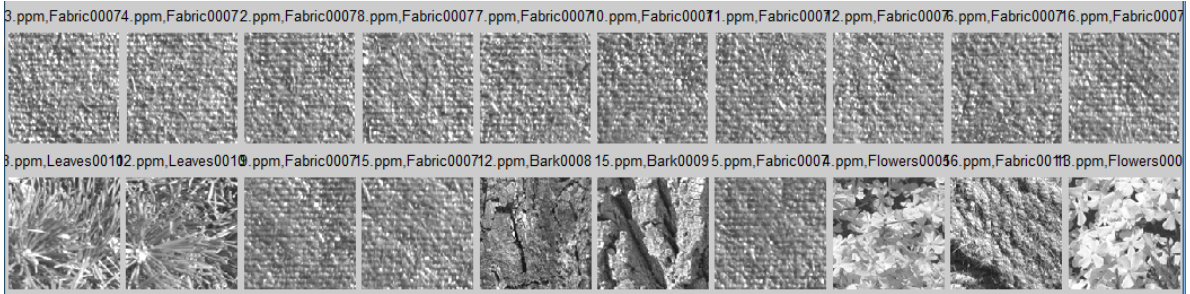

(d) GGD\&KLD+KMI method based on DT-CWT

3.ppm,Fabric00074. ppm,Fabric00072.ppm,Fabric00077.ppm,Fabric00078.ppm,Fabric000710.ppm,Fabric00071.ppm,Fabric00072.ppm,Fabric00076.ppm,Fabric00076. ppm, Fabric0007

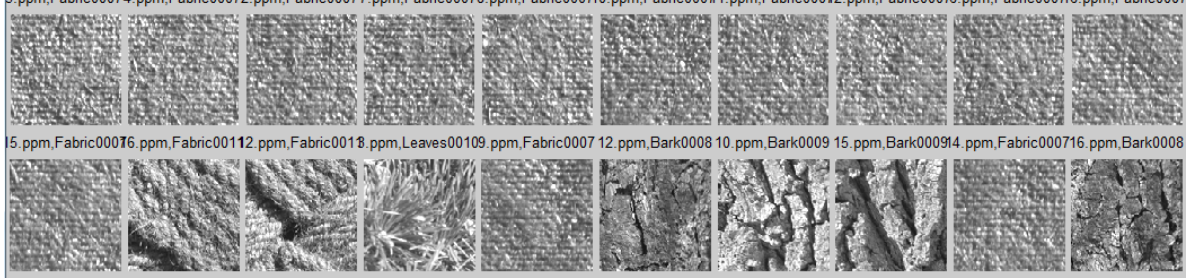

(e) GGD\&KLD+KMI method based on NSST

Fig. 2. Image retrieval example 1 


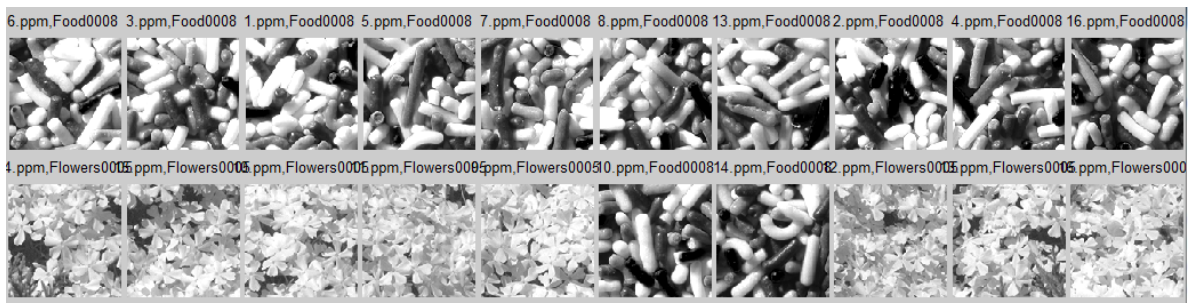

(a) GGD\&KLD+KMI method based on DWT

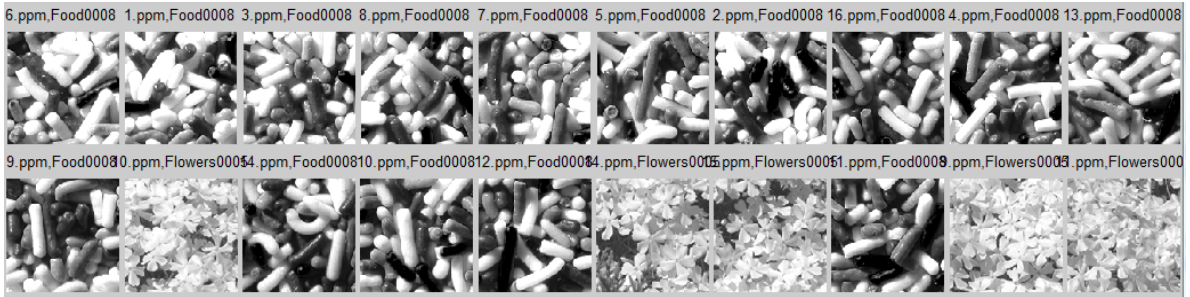

(b) GGD\&KLD+KMI method based on Coutourlet

6.ppm,Food0008 1.ppm,Food0008 3.ppm,Food0008 8.ppm,FFod0008 5.ppm,Food0008 13.ppm,Food00087.ppm,Food0008 4.ppm,FFod0008 16.ppm,Food0008 2.ppm,Food0008

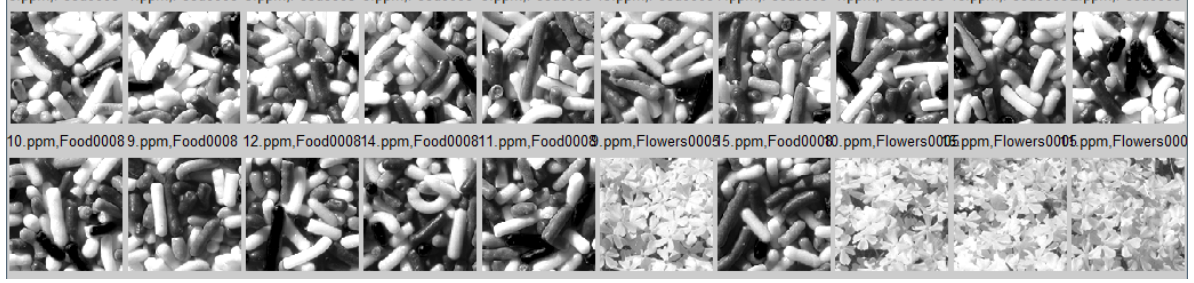

(c) GGD\&KLD+KMI method based on NSCT

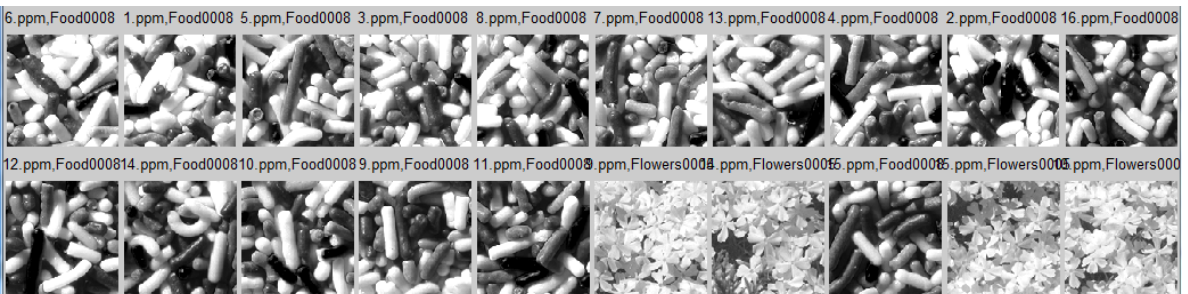

(d) GGD\&KLD+KMI method based on DT-CWT

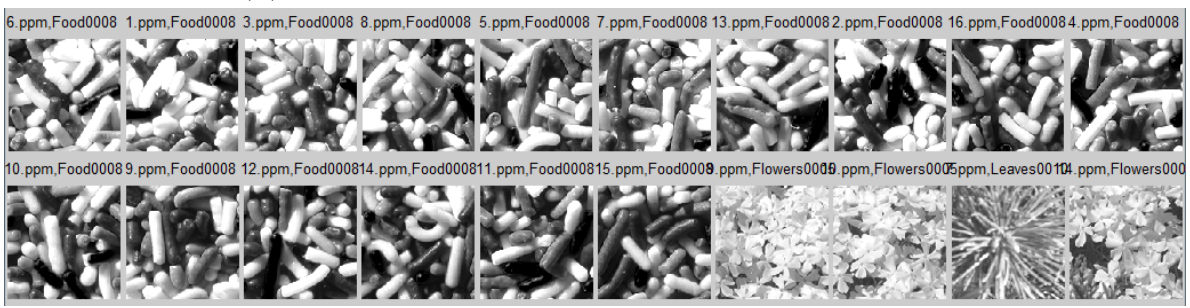

(e) GGD\&KLD+KMI method based on NSST

Fig. 3. Image retrieval example 2 


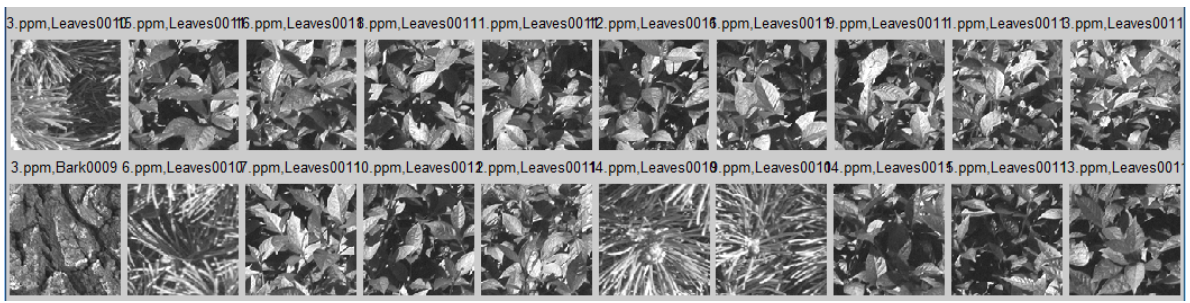

(a) GGD\&KLD+KMI method based on DWT

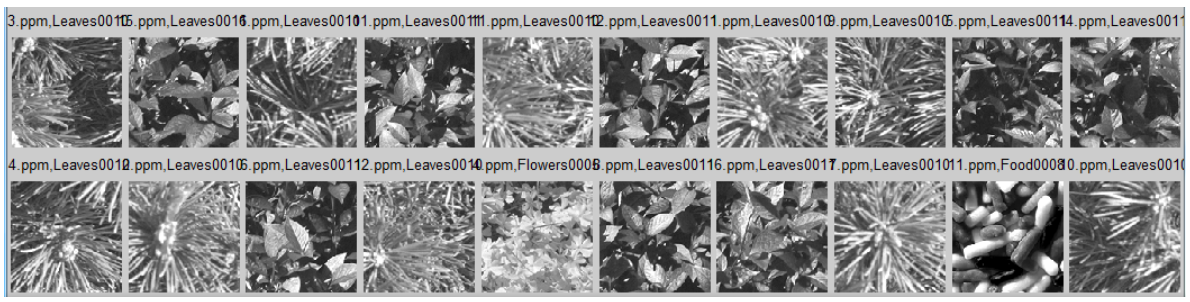

(b) GGD\&KLD+KMI method based on Coutourlet

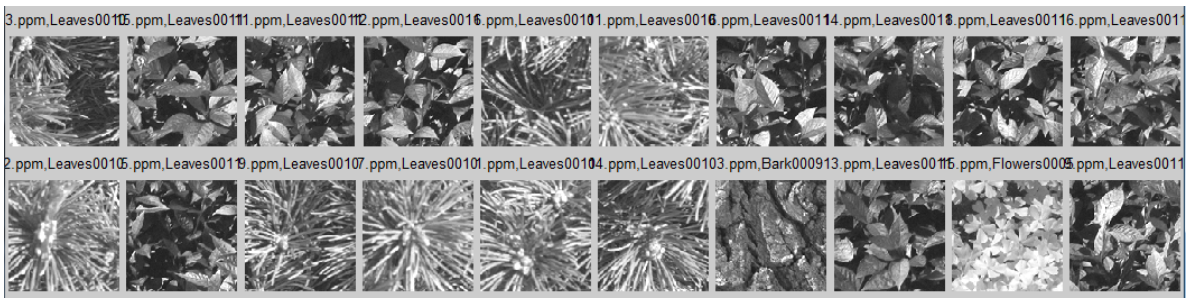

(c) GGD\&KLD+KMI method based on NSCT

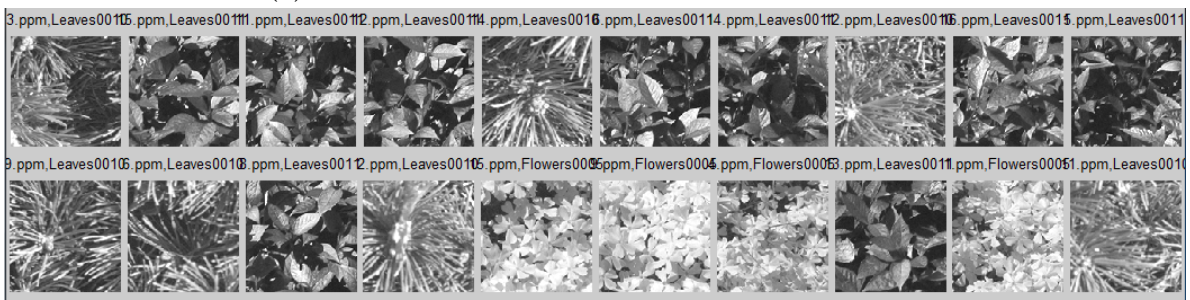

(d) GGD\&KLD+KMI method based on DT-CWT

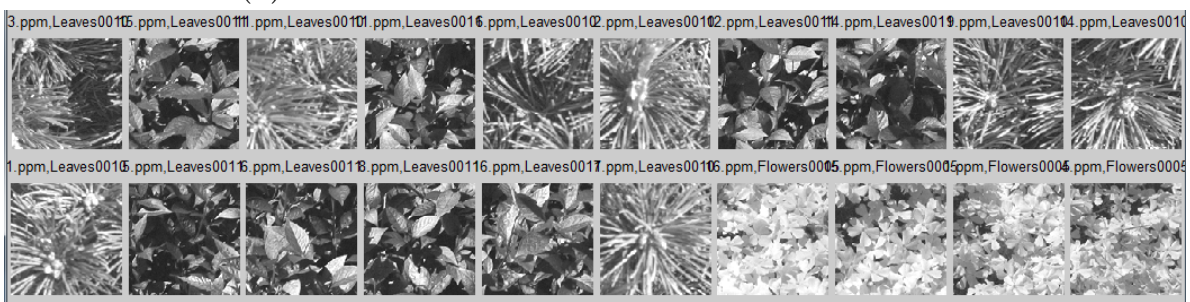

(e) GGD\&KLD+KMI method based on NSST

Fig. 4. Image retrieval example 3 


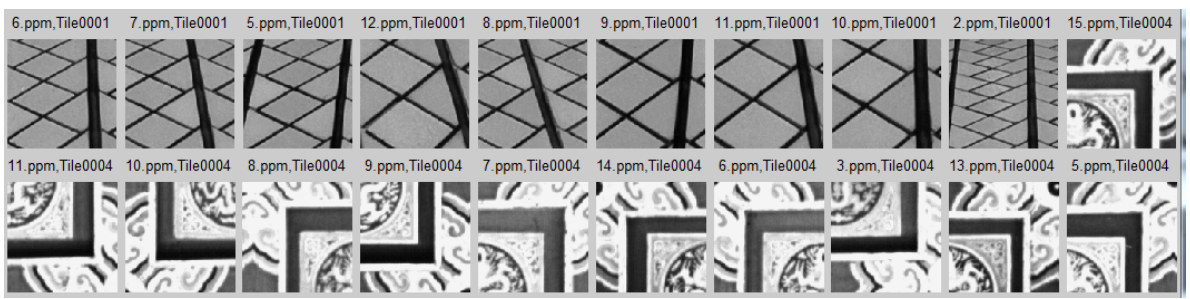

(a) GGD\&KLD+KMI method based on DWT

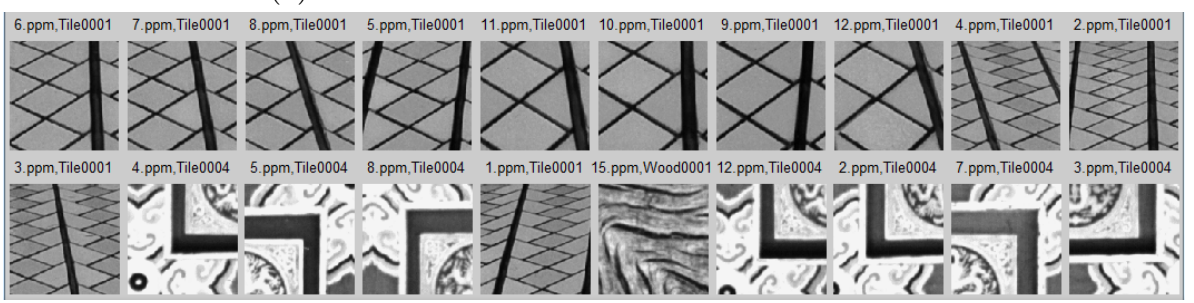

(b) GGD\&KLD+KMI method based on Coutourlet

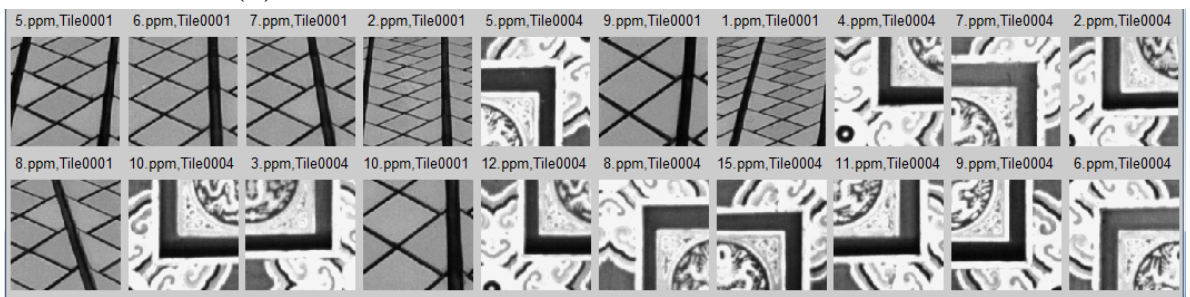

(c) GGD\&KLD+KMI method based on NSCT

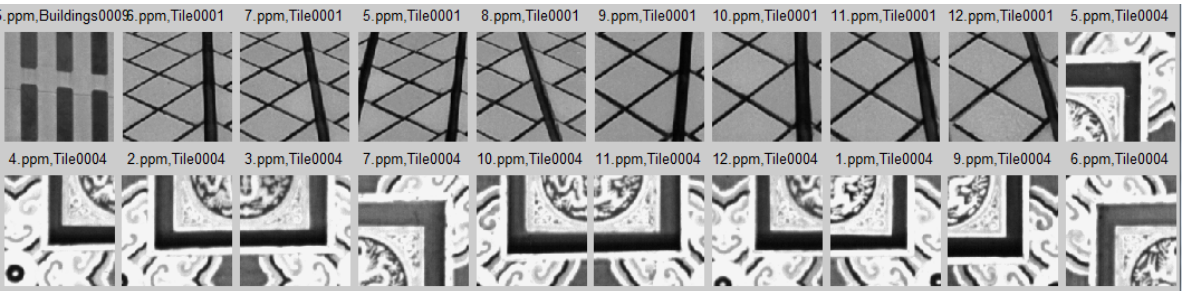

(d) GGD\&KLD+KMI method based on DT-CWT

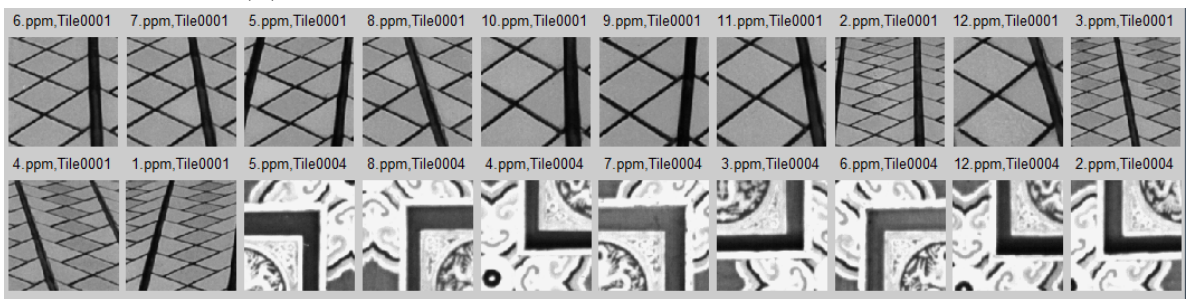

(e) GGD\&KLD+KMI method based on NSST

Fig. 5. Image retrieval example 4 


\section{References}

1. Rui Y., Huang T. S., C.S.F.: Image retrieval: Current techniques, promising directions and open issues. Journal of Visual Communication and Image Representation 10 (1999) 39-62

2. Arnold W. M. Smeulders, Marcel Worring, S.S., Amarnath Gupta, R.J.: Contentbased image retrieval at the end of the early years. IEEE Transactions on Pattern Analysis and Machine Intelligence 22 (2000) 1349-1380

3. Shandilya, S.K., Singhai, N.: Article: A survey on: Content based image retrieval systems. International Journal of Computer Applications 4 (2010) 22-26 Published By Foundation of Computer Science.

4. M. N. Do, M.V.: Wavelet-based texture retrieval using generalized gaussian density and kullback-leibler distance. IEEE Transactions on Image Processing 11 (2002) $146-158$

5. Laine, A., Fan, J.: Texture classification by wavelet packet signatures. IEEE Transactions on Pattern Recognit. Machine Intell 15 (1993) 1186-1191

6. T. Chang, C.J.K.: Texture analysis and classification with tree-structure wavelet transform. IEEE Transactions on Image Processing 2 (1993) 429-441

7. Do, M.N.: Contoulets and sparse image expansions. Proceedings of SPIE, Applications in Signal and Image Processing X 5207 (2003) 560-570

8. M. N. Do, M.V.: The contourlet transform: an efficient directional multiresolution image representation. IEEE Transactions on Image Processing 14 (2005) 20912106

9. A.L. Cunha, J.P. Zhou, M.D.: The nonsubsampled contourlet transform: theory, design and application. IEEE Trans. Image Process. 15 (2006) 3089-3101

10. D. Labate, W. Lim, G.K.: Sparse multidimensional representationusing shearlets. SPIE Proc 5914 (2005) 254-262

11. G. Easley, D. Labate, W.L.: Sparse directional image representations using the discrete shearlet transform. Appl. Comput. Harmonic Anal. 25 (2008) 25-46

12. M.K.Varanasi, B.A.: Parametric generalized gaussian density estimation. J. Acoust. Soc. Amer. 86 (1989) 1404-1415

13. D.S.Zhang: Image retrieval based on shape. Australia: Monash University (2002)

14. X. F. Ding, H. Wu, H.J.Z.: Review on shape matching. Acta Automatica Sinica 27 (2001) 678-694

15. D. S. Zhang, G.J.L.: Review of shape representation and description techniques. Pattern Recognition 37 (2004) 1-19

16. P. T. Yap, R. Paramesran, S.H.O.: Image analysis by krawtchouck moments. IEEE Transactions on Image Processing 12 (2003) 1367-1377 